\title{
Lightweight, high insertion loss, active noise enclosure with air $\operatorname{gaps}^{\mathrm{a}}$
}

\author{
C. R. Fuller and C. A. White \\ Vibration and Acoustics Laboratories, Mechanical Engineering Department, Virginia Polytechnic Institute \\ and State University, Blacksburg, Virginia 24061-0238
}

(Received 15 October 1993; revised 23 June 1994; accepted 10 October 1994)

\begin{abstract}
Preliminary experiments that demonstrate the control of noise using a lightweight, active enclosure with significant air gaps are discussed. The active enclosure consists of a lightweight, plexiglass box with piezoceramic actuators bonded to each side as active inputs. The control approach used is the filtered-x LMS. Global reductions of 7 to $20 \mathrm{~dB}$ are reported and mechanisms of control are discussed.
\end{abstract}

PACS numbers: $43.40 . \mathrm{Dx}, 43.40 . \mathrm{Vn}$

\section{INTRODUCTION}

A fundamental method of noise control is the implementation of a passive insertion loss (IL) enclosure. To be effective, this enclosure must be airtight and its walls must be of a very stiff material to successfully attenuate radiated noise from the source inside. ${ }^{1}$ These requirements establish some limitations to the use of an IL enclosure. Any device that requires a fresh air supply for cooling or intake cannot be enclosed successfully as acoustic energy would certainly leak through the air gaps in the enclosure. The requirement that the enclosure have stiff walls, which becomes even more stringent at low frequencies, reduces the practicality of IL enclosures in many applications.

Recent work in Active Structural Acoustic Control (ASAC) has suggested the possibility of an active, high insertion loss enclosure. ${ }^{2,3}$ This type of enclosure could be constructed of a lightweight, flexible material and could have air gaps in its walls for cooling, control cables, and material handling. The active enclosure would have an array of vibrational inputs attached to its walls and a computer controller would use these inputs to excite the structure of the enclosure with proper frequency, amplitude and phase distribution in such a way as to minimize radiated sound from the noise source and the enclosure combined. Waters and Bernhard have previously investigated the use of an active enclosure with air gaps. ${ }^{4}$ However in the experiments performed by Waters and Bernhard the control was achieved by an active acoustic source (a small speaker) located inside the enclosure rather than the structurally mounted vibration actuators of this study.

A lightweight, active enclosure with air gaps has been developed and tested. Some illustrative results of these experiments are given here. The enclosure was built to surround an acoustic loudspeaker which was used to simulate tonal noise from a machine. Control for the experiment was provided by a narrow-band filtered- $x$ adaptive LMS algorithm implemented on a Spectrum TMS320C25 DSP computer board optimizing signals fed to piezoceramic actuators

\footnotetext{
${ }^{a}$ This work was first presented at Noise Con 92, Williamsburg, VA, May 1992.
}

bonded to the enclosure structure. The experiment was designed to be adjustable in the sense that the amount of air gap on the top and bottom of the enclosure could be changed. The goals of the experiment were to show that the active enclosure could provide useful attenuation and to evaluate the effects of size and location of air gaps on the global attenuation levels. The technique was then briefly tested on a realistic noise source consisting of a small reciprocating compressor from a typical household refrigerator.

\section{EXPERIMENTAL SETUP}

The experimental setup, shown in the photograph of Fig. 1 , consists of the active enclosure placed over a noise source surrounded by microphones. The experiments were performed in an anechoic chamber while the controller and related electronic equipment were placed outside the chamber.

The active enclosure is box-like, constructed of $6.35-\mathrm{mm}$ acrylic sheet and measures 305 by 406 by $305 \mathrm{~mm}$ high giving a first cross mode resonance of approximately $425 \mathrm{~Hz}$. The bottom of the enclosure is open so the device can simply be set over a noise source. The panels of the enclosure are secured to each other with machine screws. This facilitates assembly and experimental modifications such as creating an air gap at the top of the enclosure by spacing it out on its screws. An air gap is created on the bottom by placing the entire enclosure on wooden blocks.

As shown in Fig. 1, piezoceramic wafer elements are bonded to each side of the panels at the center. The elements are type $\mathrm{G} 1195$ and have dimensions 38 by 63.5 by 0.178 $\mathrm{mm}$ thick. A pair of elements on a panel, when wired in parallel with 180-deg phase shift, are termed an actuator and provide uniform bending about the neutral axis of the panel. These actuators, when driven with oscillating voltage levels of up to $250 \mathrm{~V}$ peak-to-peak produce high levels of vibration in the panels and corresponding acoustic radiation from the enclosure. For the tests described here the actuators on opposite side panels of the box were wired together in phase which with the top actuator provided three independent channels of control. This arrangement was chosen due to the noise source acting as a monopole like source in the test frequencies of interest. 


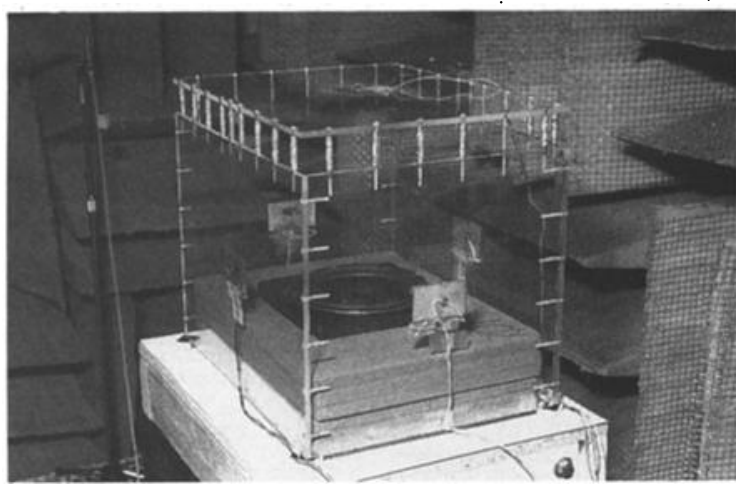

FIG. 1. Active enclosure and test arrangement.

The noise source for the experiment consists of a $165-\mathrm{mm}$ acoustical speaker mounted in a 184 by 184 by 114 $\mathrm{mm}$ high reflex speaker box. The dimensions of this speaker box occupies $10 \%$ of the inside volume of the active enclosure. The speaker was driven at a single frequency of interest.

Three $\frac{1}{2}$-in. B\&K microphones were placed 90 deg apart in a semicircle at a distance of $1.7 \mathrm{~m}$ from the center of the box, i.e., at angles of $\theta=-45^{\circ}, 0^{\circ}, 45^{\circ}$ in Figs. 2-5. These microphones serve to provide error signals for the controller. An additional microphone was mounted on a stepper motor traverse to sweep a semi-circle around the experiment at the same radius of $1.7 \mathrm{~m}$ to collect directivity data.

The controller consists of an three-channel narrow-band filtered- $x$ LMS control algorithm ${ }^{5}$ impleménted on a Spectrum TMS320C25 DSP board resident in a personal computer. Sample rates were set at $2.5 \mathrm{kHz}$, while the reference input to the controller was taken directly from the signal driving the noise source. Two coefficients were used in each adaptive filter.

\section{EXPERIMENTAL PROCEDURE}

The tests described here were performed at two frequencies, 200 and $450 \mathrm{~Hz}$ which respectively correspond to well below and near the box first cross mode resonance frequency. In each test the noise source was driven with a 1-Vrms signal fed into the amplifier before control. First, the acoustic ra-

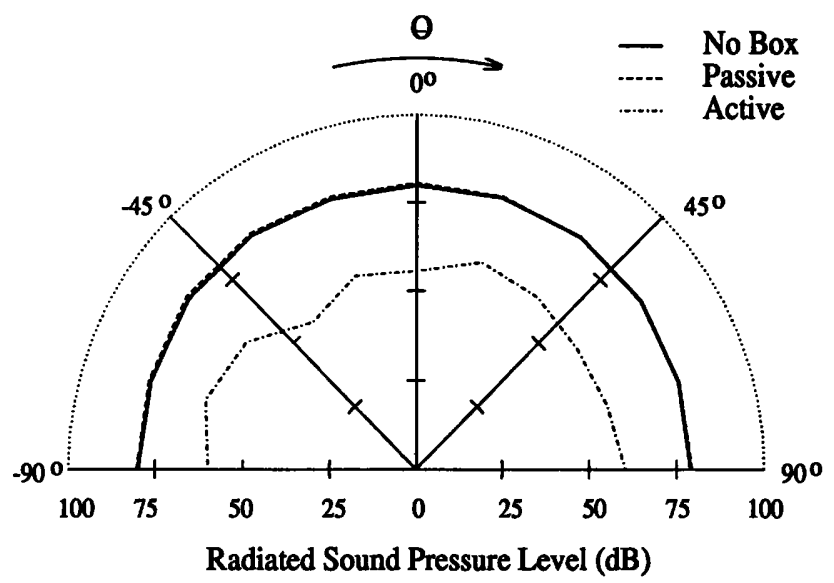

FIG. 2. Radiation directivity, $f=200 \mathrm{~Hz}, 50.8-\mathrm{mm}$ bottom air gap.

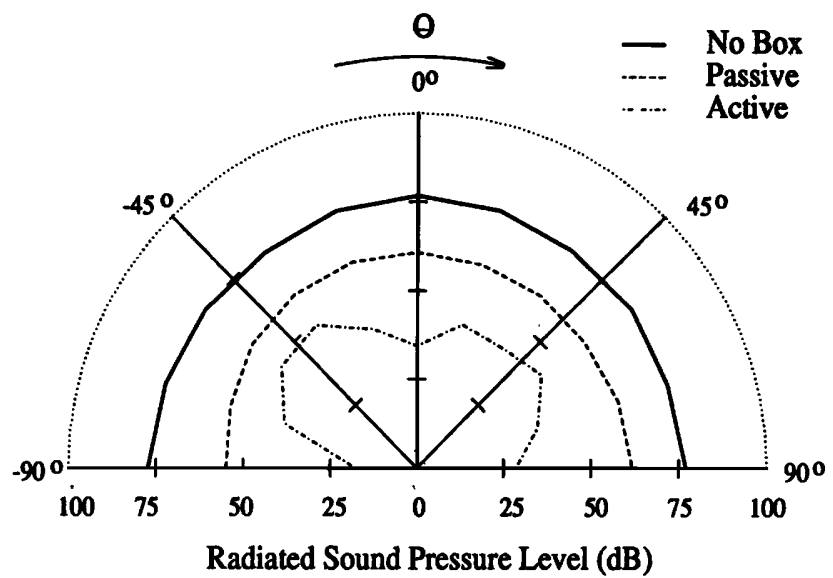

FIG. 3. Radiation directivity, $f=450 \mathrm{~Hz}, 50.8-\mathrm{mm}$ bottom air gap.

diation directivity of the noise source by itself was measured over a semi-circle. The active IL box was then placed over the source and the acoustic radiation was measured without control to obtain the amount of passive attenuation caused by the box structure. The controller was then used to minimize the error microphones by adjusting the complex voltage to the piezoceramic actuators and the radiation directivity was again measured. The top and bottom air gaps were changed in configuration from test to test to determine their effect on the performance of the active enclosure.

\section{EXPERIMENTAL RESULTS}

Results are now given for two configurations each with large gaps around the enclosure. The first configuration has a $50.8-\mathrm{mm}$ gap on the bottom of the enclosure providing a $13 \%$ open area of the enclosure surface. The second has only a 12.7-mm gap on the bottom but a $50.8-\mathrm{mm}$ gap on the top corresponding to $16 \%$ open area. With the enclosure in a particular configuration, tests were performed at each frequency. Results are given in Figs. 2-5 as sound pressure level directivity versus $\theta$, the sweep angle of the traverse sampled at every $18^{\circ}$.

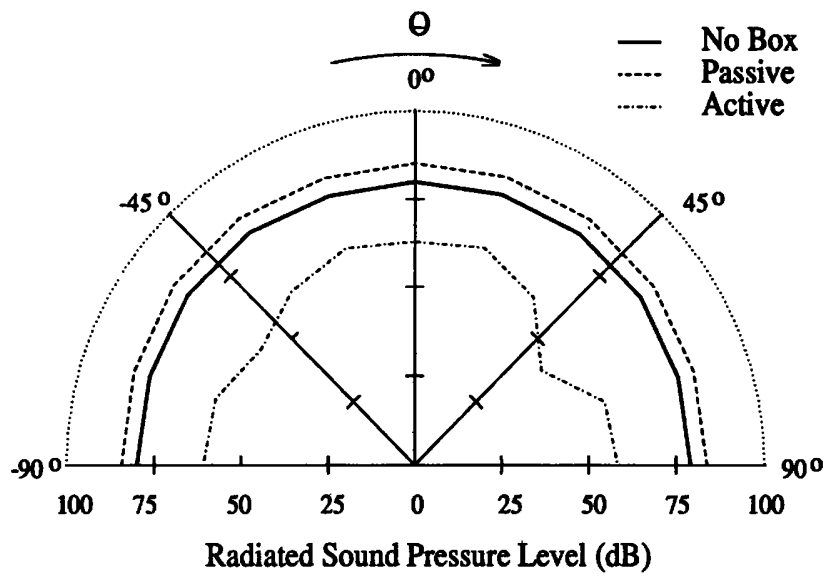

FIG. 4. Radiation directivity, $f=200 \mathrm{~Hz}, 12.7-\mathrm{mm}$ bottom and 50.8-mm top air gaps. 


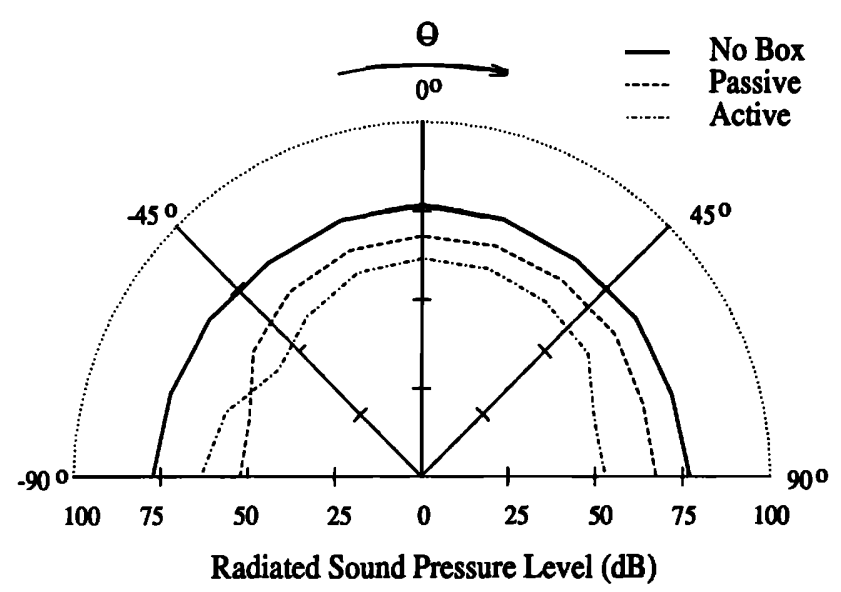

FIG. 5. Radiation directivity, $f=450 \mathrm{~Hz}, 12.7-\mathrm{mm}$ bottom and 50.8-mm top air gaps.

Figure 2 shows acoustic directivity levels at $200 \mathrm{~Hz}$ with the $50.8-\mathrm{mm}$ gap at the bottom. The box by itself provides very little passive attenuation. With control however, almost $20 \mathrm{~dB}$ of attenuation is achieved. In the same configuration at $450 \mathrm{~Hz}$, Fig. 3, the box provides roughly $15 \mathrm{~dB}$ of passive attenuation and control adds another $20 \mathrm{~dB}$. Notice that at 0 , 90 , and -90 deg the directivity of Fig. 3 is notched because the controller minimized the error microphones near these locations. This suggests that at this higher frequency, multiple modes of the box structure are contributing to the radiation. For both these tests, measurements out of the traverse plane give similar attenuation levels confirming the noise reduction was global.

In the second configuration, with a large air gap at the top and a smaller gap at the bottom of the enclosure, the results are somewhat different. At $200 \mathrm{~Hz}$ the enclosure actually increases the acoustic radiation by $5 \mathrm{~dB}$, Fig. 4, most likely due to internal resonance phenomena. When control is activated, the radiation is reduced $20 \mathrm{~dB}$ providing an overall attenuation of about $15 \mathrm{~dB}$. Figure 5 shows the results for the same test configuration at $450 \mathrm{~Hz}$. Here, the enclosure provides 5- to 7-dB passive attenuation and another 5-7 dB active for a total of $15 \mathrm{~dB}$. The crossing of the uncontrolled and controlled directivity plots near $-90 \mathrm{deg}$ is due to multimodal response of the enclosure and spill-over associated with the multi-channel controller. Again additional measurements confirm the attenuation is global.

It is interesting to postulate on the mechanisms of control. It is likely that the active inputs control and modify the vibration of the panels (but do not totally cancel their vibration) so that the sound transmission through them effectively cancels the acoustic radiation due to sound leakage through the air gaps. This behavior is similar to what has been previously termed "modal restructuring." It is also likely that the size of the interior acoustic space is important. Smaller acoustic spaces will lead to a higher fundamental resonance frequency and thus more uniform monopole like response of the contained acoustic space at the driving frequency of interest. Thus the box can be constructed to act as a "filter" that can effectively reduce the source order and make the control task easier. However if the space is to small and the

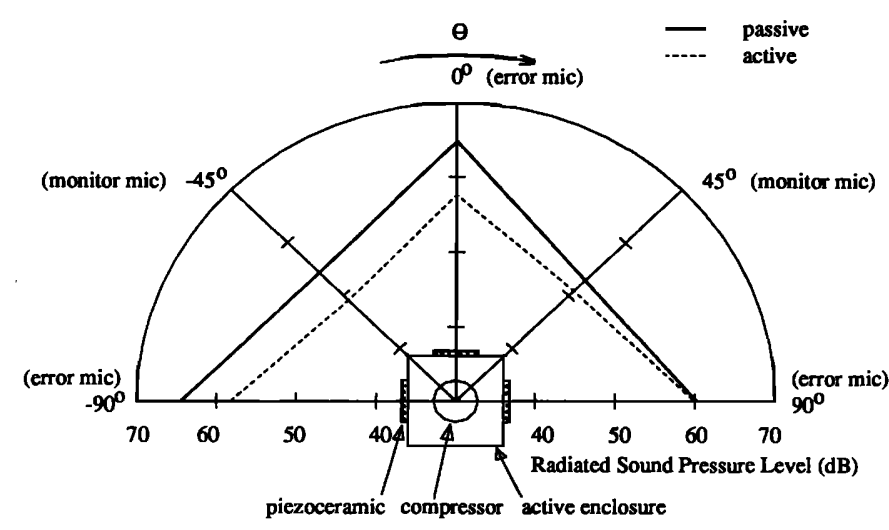

FIG. 6. Radiation directivity, overall sound pressure level, $25-\mathrm{mm}$ bottom and 6-mm top air gaps.

active surfaces are very close to the noise source then the active inputs will be effectively directly controlling the vibration of the internal source itself, negating the effect of the box. These important mechanisms need further study, particularly for optimal design of the system.

\section{PRACTICAL DEMONSTRATION}

In order to test the practicality of the technique, a brief experiment was performed using a small reciprocating compressor from a typical household refrigerator as a realistic noise source. Masters et al. ${ }^{7}$ have carried out related experiments in which a piezoceramic actuator was bonded directly to the shell of a similar compressor and used to actively control the sound radiation. The approach of Masters $e t$ al. thus relies on directly controlling or modifying the source vibration to reduce the sound radiation and with the single actuator, only localized reduction of sound was obtained.

The characteristics of the noise produced by the compressor were that the radiation had a dipole-like pattern in the plane of the compressor (due to the motion of the internal pistons) and the spectral content was dominated by pure tones at harmonics of near $58,116,174$, and $232 \mathrm{~Hz}$, etc. The experimental configuration was varied slightly for this test. The active enclosure used was of dimensions 300 by 288 by $248 \mathrm{~mm}$ high with a $6 \mathrm{~mm}$ and $25 \mathrm{~mm}$ top and bottom air gap, respectively, giving an open area of $9 \%$. Three independent active inputs were applied to the piezoceramic actuators on three vertical sides of the box due to the more complicated form of the noise source as shown in Fig. 6. The controller was re-configured so that the reference signal was taken from an accelerometer mounted directly on the compressor casing. The adaptive controller was modified so that each adaptive filter contained 25 coefficients while the fixed filters had 60 coefficients. The sample rate used was $1.8 \mathrm{kHz}$. Three error microphones opposite each active surface as shown in top view of Figure 6 were used while two additional monitor microphones were placed at $\theta= \pm 45^{\circ}$.

Figure 6 presents the overall sound pressure levels for the bandwidth 0 to $800 \mathrm{~Hz}$ measured at the five microphones and plotted as a radiation directivity pattern for the cases with the box in place (passive) and the control turned on (active). The box itself was found to provide negligible pas- 
sive attenuation of the noise source. The results show attenuations of up to $7 \mathrm{~dB}$ in total sound pressure level across the bandwidth. Measurements outside of the microphone plane of Fig. 6 confirm that this trend is global. The use of the active control also results in a noticeable audible reduction in sound as there were no significant harmonics at frequencies greater than $800 \mathrm{~Hz}$. The test thus confirms that the active enclosure performs adequately on realistic sources.

\section{CONCLUSIONS}

An active insertion loss enclosure has been developed and tested. It has been demonstrated that the enclosure may be constructed of a lightweight, flexible material and have provisions for airflow through its walls. In each test configuration at least $15 \mathrm{~dB}$ of global attenuation is achieved at different frequencies for an ideal noise source. Reductions of $7 \mathrm{~dB}$ in overall sound pressure level were measured with a realistic noise source consisting of a small reciprocating compressor. Additional experiments show that if the enclosure is optimally configured by changing system parameters such as internal volume size, number of control channels, and configuration of piezoelectric actuators even more attenuation is possible. It is suspected that attenuation occurs due to both control of transmission through the panels by increasing their stiffness and interaction of the panel radiation with sound propagating through the enclosures air gaps. These phenomena need further investigation.

The active portion of the enclosure is created by vibrational inputs to the walls adjusted by an LMS controller so as to minimize sound radiation (ASAC). It has been shown here that piezoceramic actuators are acceptable vibrational inputs for such an active enclosure.
The work reported in this letter demonstrates that the active noise enclosure shows promise in applications where the vibrational characteristics of a machine cannot be easily controlled and a provision for airflow, piping, or cabling must be made. Further work is needed in testing on realistic machines and optimization of system parameters.

\section{ACKNOWLEDGMENTS}

The authors gratefully acknowledge support of this work by Noise Cancellation Technologies, Linthicum, MD.

${ }^{1}$ L. L. Beranek, Noise and Vibration Control (McGraw-Hill, New York, 1971).

${ }^{2}$ C. R. Fuller, "Apparatus and Method for Global Noise Reduction," U.S. Patent No. 4,715,599 (1987).

${ }^{3}$ C. R. Fuller, C. H. Hansen, and S. D. Snyder, "Experiments on Active Control of Sound Radiation from a Panel Using a Piezoceramic Actuator," J. Sound Vib. 150(2), 179-190 (1991).

${ }^{4}$ D. E. Waters and R. J. Bernhard, "Enhancement of Close Fitting Enclosure Performance with Active Noise Control," Proc. Inter-Noise 89, pp. 463-466, Newport Beach, CA (December 1989).

${ }^{5}$ S. J. Elliott, I. M. Stothers, and P. A. Nelson, "A Multiple Error LMS Algorithm and its Application to the Active Control of Sound and Vibrations," IEEE Trans. Acoust. Speech Signal Process. ASSP-35(1), 14241434 (1987).

${ }^{6}$ C. R. Fuller, C. H. Hansen, and S. D. Synder, "Experiments on Active Control of Sound Radiation from a Vibrating Rectangular Panel by Sound Sources and Vibration Inputs: An Experimental Comparison," J. Sound Vib. 145(2), 195-215 (1991).

${ }^{7}$ A. R. Masters, S. J. Kim, and J. D. Jones, "Experimental Investigation Into Active Control of Compressor Noise Radiation Using Piezoelectric Actuators," Proc. Inter-Noise 92, pp. 395-400, Toronto, Canada (July 1992). 